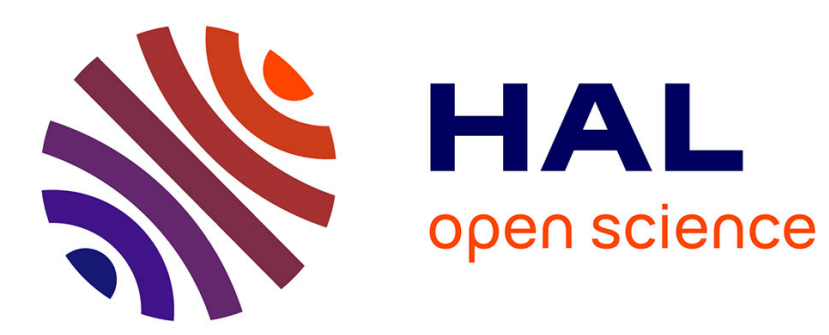

\title{
AUTOMORPHISMS AND DYNAMICS: A LIST OF OPEN PROBLEMS
}

\author{
Serge Cantat
}

\section{To cite this version:}

Serge Cantat. AUTOMORPHISMS AND DYNAMICS: A LIST OF OPEN PROBLEMS. International Congress of Mathematicians (ICM), 2018, Rio de Janeiro, Brazil. hal-02334183

\section{HAL Id: hal-02334183 \\ https://hal.science/hal-02334183}

Submitted on 25 Oct 2019

HAL is a multi-disciplinary open access archive for the deposit and dissemination of scientific research documents, whether they are published or not. The documents may come from teaching and research institutions in France or abroad, or from public or private research centers.
L'archive ouverte pluridisciplinaire HAL, est destinée au dépôt et à la diffusion de documents scientifiques de niveau recherche, publiés ou non, émanant des établissements d'enseignement et de recherche français ou étrangers, des laboratoires publics ou privés. 


\title{
AUTOMORPHISMS AND DYNAMICS: A LIST OF OPEN PROBLEMS
}

\author{
SERGE CANTAT
}

\begin{abstract}
We survey a few results concerning groups of regular or birational transformations of projective varieties, with an emphasis on open questions concerning these groups and their dynamical properties.

AMS Classification. Primary 14J50, 37F99 ; Secondary 14E07, 14J26, 14J28, 14J32, 14P25, 32M05, 37E30.

KEY WORDS. Projective varieties, groups of automorphisms, transformation groups, complex dynamics, birational geometry.
\end{abstract}

\section{INTRODUCTION}

1.1. Algebraic transformations. Let $\mathbf{k}$ be a field and $d$ be a positive integer. Consider a smooth projective variety $X_{\mathbf{k}}$ of dimension $d$, defined over the field $\mathbf{k}$. Attached to $X_{\mathbf{k}}$ are two groups of algebraic transformations: its group of birational transformations $\operatorname{Bir}\left(X_{\mathbf{k}}\right)$ and the subgroup of (biregular) automorphisms $\operatorname{Aut}\left(X_{\mathbf{k}}\right)$. An element $f$ of $\operatorname{Bir}\left(X_{\mathbf{k}}\right)$ is defined by its graph $\mathfrak{G}_{f} \subset X_{\mathbf{k}} \times X_{\mathbf{k}}$; by definition, $\mathfrak{G}_{f}$ is an irreducible algebraic subvariety of dimension $d$ such that the two projections $\mathfrak{G}_{f} \rightarrow X_{\mathbf{k}}$ have degree one, and in the case of automorphisms, the two projections are isomorphisms (in particular, they do not contract any algebraic subset of positive dimension onto a point).

For simplicity, unless otherwise specified, we assume that $\mathbf{k}=\mathbf{C}$ is the field of complex numbers. We denote by $X$ the variety, with no reference to its field of definition, and by $X(\mathbf{C})$ its complex points; thus, $X(\mathbf{C})$ is also a compact, complex manifold of dimension $d$ (and of real dimension $2 d$ ). The group $\operatorname{Bir}(X)$ coincides with the group of bimeromorphic transformations of $X(\mathbf{C})$, and $\operatorname{Aut}(X)$ coincides with the subgroup of holomorphic diffeomorphisms.

A birational transformation $f: X \rightarrow X$ is a pseudo-automorphism if there exist two Zariski closed subsets $Z$ and $Z^{\prime}$ of codimension $\geq 2$ in $X$ such that $f$ induces an isomorphism from $X \backslash Z$ to $X \backslash Z^{\prime}$. Equivalently, $f$ and its inverse $f^{-1}$ do not contract any hypersurface (they are "isomorphisms in codimension 1"). Pseudo-automorphisms constitute an important subgroup $\operatorname{Psaut}(X)$ of $\operatorname{Bir}(X)$ that contains $\operatorname{Aut}(X)$. As we shall see, automorphisms are quite rare in dimension $\geq 3$;

The author is grateful to the CNRS, Université Rennes 1, and foundation del Duca from the french Academy of Sciences, for their support, and expresses his warmest thanks to Eric Bedford, Jérémy Blanc, Yves de Cornulier, Julie Déserti, Jeffrey Diller, Igor Dolgachev, Romain Dujardin, Charles Favre, Vincent Guirardel, Stéphane Lamy, Federico Lo Bianco, Bjorn Poonen, Juan Souto, and Christian Urech for interesting discussions on the topics covered in this survey. 
pseudo-automorphisms appear more frequently, for instance in the study of some special varieties, such as rational varieties and Calabi-Yau varieties.

\subsection{Examples.}

1.2.1. Projective spaces. Consider the projective space $\mathbb{P}_{\mathbf{k}}^{d}$ of dimension $d$. Its group of automorphisms is the group of linear projective transformations $\mathrm{PGL}_{d+1}(\mathbf{k})$. Its group of birational transformations is known as the Cremona group in $d$ variables. In homogeneous coordinates $\left[x_{1}: \ldots: x_{d+1}\right]$, every birational transformation $f$ of $\mathbb{P}_{\mathbf{k}}^{d}$ can be written as

$$
f\left[x_{1}: \ldots: x_{d+1}\right]=\left[f_{1}: \ldots: f_{d+1}\right]
$$

where the $f_{i}$ are homogeneous polynomials in the variables $x_{i}$, of the same degree, and without common factor of positive degree. In the affine coordinates $X_{i}=x_{i} / x_{d+1}$, it is defined by rational fractions

$$
F_{i}\left(X_{1}, \ldots, X_{d}\right)=\frac{f_{i}\left(X_{1}, \ldots, X_{d}, 1\right)}{f_{d+1}\left(X_{1}, \ldots, X_{d}, 1\right)} .
$$

When $d=1, \operatorname{Bir}\left(\mathbb{P}_{\mathbf{k}}^{1}\right)$ coincides with the group of automorphisms $\mathrm{PGL}_{2}(\mathbf{k})$. When $d \geq 2, \operatorname{Bir}\left(\mathbb{P}_{\mathbf{k}}^{d}\right)$ is much bigger than $\mathrm{PGL}_{d+1}(\mathbf{k})$. In dimension 2 , it contains all monomial transformations

$$
\left(X_{1}, X_{2}\right) \mapsto\left(X_{1}^{a} X_{2}^{b}, X_{1}^{c} X_{2}^{b}\right)
$$

with $a d-b c= \pm 1$, all transformations $\left(X_{1}, X_{2}\right) \mapsto\left(X_{1}, X_{2}+h\left(X_{1}\right)\right)$, for every $h \in \mathbf{k}\left(X_{1}\right)$, all linear projective transformations, hence all compositions of such maps. When $\mathbf{k}=\mathbf{C}$, the Hénon map

$$
\left(X_{1}, X_{2}\right) \mapsto\left(X_{2}, X_{1}+X_{2}^{2}+c\right)
$$

provides a transformation of the plane with a rich dynamics (its topological entropy is equal to $\log (2)$ for every parameter $c$, it has infinitely many periodic points, ...).

1.2.2. Abelian varieties. Next, consider an elliptic curve $E=\mathbf{C} / \Lambda$, where $\Lambda$ is a lattice in $\mathbf{C}$. The product $A=E^{d}$ is a complex torus of dimension $d$; it is also a projective variety, hence an example of an abelian variety. The group of birational transformations of $A$ coincides with its group of automorphisms. It contains the group $A$ itself, acting by translations, as well as the group $\mathrm{GL}_{d}(\mathbf{Z})$, acting by linear transformations on $A$ (or more precisely on its universal cover $\mathbf{C}^{d}$, preserving the lattice $\Lambda^{d}$, hence also on $A$ after taking the quotient by $\Lambda^{d}$ ).

1.2.3. Calabi-Yau varieties. As a third example, fix an integer $d \geq 1$, and consider a smooth hypersurface $X$ in $\left(\mathbb{P}_{\mathbf{C}}^{1}\right)^{d+1}$ which is defined in the open set $\mathbf{C}^{d+1}$ of $\mathbb{P}^{1}(\mathbf{C})^{d+1}$ by a polynomial equation $P\left(x_{1}, \ldots, x_{d+1}\right)=0$ whose degree is equal to 2 with respect to each coordinate $x_{j}$. Geometrically, this means that for every index $1 \leq i \leq d+1$, the projection $\pi_{i}: X \rightarrow\left(\mathbb{P}_{\mathbf{C}}^{1}\right)^{d}$ which forgets the $i$-th coordinate is a morphism of degree 2. The involution that permutes the two points in the fibers of $\pi_{i}$ is a birational involution $\sigma_{i}$ of $X$.

When $d \geq 2$, these hypersurfaces of degree $(2,2, \ldots, 2)$ in $\left(\mathbb{P}_{\mathbf{C}}^{1}\right)^{d+1}$ are examples of Calabi-Yau varieties: they are simply connected, they support a holomorphic 
$d$-form that does not vanish, and they do not split as the product of two varieties of smaller dimension. Thus, the involutions $\sigma_{i}$ are pseudo-automorphisms because $\operatorname{Bir}(X)=\operatorname{Psaut}(X)$ for all Calabi-Yau varieties, as one sees by pulling back a nonvanishing holomorphic $d$-form.

In dimension $\leq 2$, these pseudo-automorphisms are in fact regular automorphisms. In dimension $1, X$ is a curve of genus 1 , and the involutions determine a dihedral group, acting by affine transformations of type $z \mapsto \pm z+a$ on the elliptic curve. In dimension $d=2, X$ is a $\mathrm{K} 3$ surface, and the involutions determine a large group of automorphisms of $X$ : if the equation $P$ is generic, then $\operatorname{Aut}(X)=\operatorname{Bir}(X)$ is generated by those three involutions, there are no relations between these involutions (they generate a group isomorphic to the free product $\mathbf{Z} / 2 \mathbf{Z} \star \mathbf{Z} / 2 \mathbf{Z} \star \mathbf{Z} / 2 \mathbf{Z}$ ), and the composition $\sigma_{1} \circ \sigma_{2} \circ \sigma_{3}$ has a rich dynamical behaviour (its topological entropy is $\log (9+4 \sqrt{5}))$.

For $d \geq 3$, the involutions $\sigma_{i}$ are pseudo-automorphisms with indeterminacy points. For a generic choice of the equation $P$, the involutions $\sigma_{i}$ generate $\operatorname{Psaut}(X)$, there are no relations between the $\sigma_{i}$, and $\operatorname{Aut}(X)$ is trivial (see [13]).

1.3. Plan. Our goal is to review some important facts concerning the group $\operatorname{Aut}(X)$, the algebraic structure of its subgroups, and the dynamical properties of its elements. The emphasis is on open problems, in which algebraic geometry, group theory, and dynamics are simultaneously involved.

This article comprises two main parts. The first one concerns groups of automorphisms of smooth complex projective varieties, and their action on the cohomology of the variety. The second part concerns the dynamics: we focus on automorphisms with a dynamical behavior of low complexity because their study has been surprisingly neglected, while it offers interesting questions at the interface between dynamics and algebraic geometry.

We focus on $\operatorname{Aut}(X)$ for simplicity. As Section 1.2.3 shows, it would be better to work with pseudo-automorphisms, or even with birational transformations. In fact, most of the questions which are described below could be stated for pseudoautomorphisms of compact kähler manifolds (see Remark 2.1); and some of them concern birational transformations of projective varieties over an arbitrary field.

\section{Contents}

1. Introduction 1

2. Groups of automorphisms 3

3. Dynamics with low complexity 8

References

\section{Groups of Automorphisms}

2.1. Automorphisms. Let $X$ be a smooth complex projective variety. Its group of automorphisms is a complex Lie group (for the topology of uniform convergence), whose Lie algebra is the finite dimensional algebra of regular vector fields on $X$. 
$\operatorname{But} \operatorname{Aut}(X)$ may have infinitely many components, as shown in Section 1.2.2 by the example of the abelian variety $(\mathbf{C} / \Lambda)^{d}$, where $\Lambda \subset \mathbf{C}$ is a lattice and $d \geq 2$.

Consider the action of $\operatorname{Aut}(X)$ on the cohomology $H^{*}(X ; \mathbf{Z})$ of $X(\mathbf{C})$; this gives a linear representation

$$
\operatorname{Aut}(X) \rightarrow \mathrm{GL}\left(H^{*}(X ; \mathbf{Z})\right) .
$$

The connected component of the identity $\operatorname{Aut}(X)^{0} \subset \operatorname{Aut}(X)$ acts trivially on the cohomology, and is therefore contained in the kernel of this representation. If an element $f \in \operatorname{Aut}(X)$ acts trivially on the second cohomology group $H^{2}(X ; \mathbf{Z})$, it preserves the cohomology class of a kähler form $\kappa$ on $X$ (resp. the first Chern class of an ample line bundle $L$ on $X$ ); this means that the volume (resp. degree) of the graph of $f$ is uniformly bounded on the kernel of the representation (5). Since subvarieties with a fixed volume (resp. degree) form a bounded family, one can use Douady spaces (or Hilbert Schemes) to obtain the following fact: the connected component of the identity in $\operatorname{Aut}(X)^{0} \subset \operatorname{Aut}(X)$ has finite index in the kernel of the linear representation $\operatorname{Aut}(X) \rightarrow \mathrm{GL}\left(H^{2}(X ; \mathbf{Z})\right.$ ) (see [31, 35] for a proof).

Thus, the group $\operatorname{Aut}(X)$ splits into two basic parts: its neutral component $\operatorname{Aut}(X)^{0}$, and its discrete image

$$
\operatorname{Aut}(X)^{*} \subset \mathrm{GL}\left(H^{*}(X ; \mathbf{Z})\right) .
$$

The group of connected components $\operatorname{Aut}(X) / \operatorname{Aut}(X)^{0}$ is an extension of $\operatorname{Aut}(X)^{*}$ by a finite group.

Remark 2.1. One may replace $H^{2}(X ; \mathbf{Z})$ by the subgroup $\mathrm{N}^{1}(X)$ generated by cohomology classes of algebraic hypersurfaces of $X$ (or more precisely by the Poincaré dual of their homology classes). The kernel of the representation Aut $(X) \mapsto \mathrm{GL}\left(\mathrm{N}^{1}(X)\right)$ is, again, equal to a finite extension of $\operatorname{Aut}(X)^{0}$. Doing so, it is possible to phrase some of the following questions for varieties which are defined over fields of arbitrary characteristic.

One may also replace $\operatorname{Aut}(X)$ by $\operatorname{Psaut}(X)$. Indeed, since pseudo-automorphisms are isomorphisms in codimension 1 , the group Psaut $(X)$ acts linearly on $\mathrm{N}^{1}(X)$ (and $H^{2}(X ; \mathbf{Z})$ when $\mathbf{k}=\mathbf{C}$ ). Thus, all questions concerning the action of $\operatorname{Aut}(X)$ on $H^{2}(X ; \mathbf{Z})$ can be stated for the action of $\operatorname{Psaut}(X)$ on $\mathrm{N}^{1}(X)$ (even when the characteristic of $\mathbf{k}$ is positive).

2.2. The realization problem. Two main problems arise: given a connected algebraic group $G$, does there exist a projective variety $X$ such that $\operatorname{Aut}(X)^{0}$ is isomorphic to $G$ as an algebraic group ? Given a subgroup $\Gamma$ of $\mathrm{GL}_{n}(\mathbf{Z})$, for some $n \geq 2$, does there exist a projective variety $X$ and an isomorphism of groups $\Gamma \simeq \operatorname{Aut}(X)^{*}$ ? There is also a third, less interesting problem, which asks which pairs $(G, \Gamma)$ may be simultaneously realized as the connected component and the discrete part of $\operatorname{Aut}(X)$, for some variety $X$.

The first problem has been solved by Brion in the following strong sense: any connected algebraic group $G$ over a perfect field is the neutral component of the automorphism group scheme of some normal projective variety $X$; if the characteristic of the field is 0 , one can moreover assume that $X$ is smooth of dimension $\operatorname{dim}(X)=2 \operatorname{dim}(G)$ (see [4]; see also [45] for Kobayashi hyperbolic manifolds).

In the following paragraphs, we focus on the discrete, countable group $\operatorname{Aut}(X)^{*}$. 
2.3. Linear groups and their Zariski closure. Not much has been proven yet concerning the description of the groups $\operatorname{Aut}(X)^{*}$. To simplify the exposition, we shall say that a group $\Gamma$ is abstractly realizable as a group of automorphisms (in dimension $d$ ) if there exist a projective variety $X$ (of dimension $d$ ) such that $\operatorname{Aut}(X)$ is isomorphic to $\Gamma$ (as abstract groups).

Remark 2.2. The group $\mathrm{GL}_{n}(\mathbf{Z})$ acts on the abelian variety $(\mathbf{C} / \Lambda)^{n}$ for every lattice $\Lambda \subset$ C. Blowing-up the origin, one gets a new variety $X$ with $\operatorname{Aut}(X)^{0}=\left\{\operatorname{id}_{X}\right\}$ and with $\mathrm{GL}_{n}(\mathbf{Z}) \simeq \operatorname{Aut}(X)$ if $\Lambda$ is generic. Thus, every subgroup $\Gamma$ of $\mathrm{GL}_{n}(\mathbf{Z})$ acts faithfully on a projective variety of dimension $n$ whose group of automorphisms is discrete. This does not say that $\Gamma$ is abstractly realizable.

Remark 2.3. A necessary condition for a countable group $\Gamma$ to be realizable is that $\Gamma$ admits a linear integral representation $\Gamma \rightarrow \mathrm{GL}_{m}(\mathbf{Z})$ with finite kernel. This is not a sufficient condition: when $n \geq 2$, there is a finite, cyclic, and central extension of the symplectic group $\mathrm{Sp}_{2 n}(\mathbf{Z})$ that does not act faithfully by birational transformations on any complex projective variety (see [14]).

Instead of looking at the abstract notion of realizability, one may also add some rigidity in the definition; this may be done as follows. Say that a group $\Gamma \subset \mathrm{GL}_{m}(\mathbf{Z})$ is strongly realizable as a group of automorphisms if there is a smooth complex projective variety $X$, and a linear algebraic representation

$$
\varphi: \mathrm{GL}_{m}(\mathbf{R}) \rightarrow \mathrm{GL}\left(H^{2}(X ; \mathbf{R})\right)
$$

which is defined over $\mathbf{Z}$, such that $\operatorname{Aut}(X)^{*}$ coincides with $\varphi(\Gamma)$. As in Remark 2.1, one may replace $H^{2}(X ; \mathbf{Z})$ by $\mathrm{N}^{1}(X)$ to define a notion of strong realizability by pseudo-automorphisms, defined over any field $\mathbf{k}$.

Remark 2.4. The cohomology group $H^{2}(X ; \mathbf{C})$ splits into the direct sum of the Dolbeault groups $H^{p, q}(X ; \mathbf{C})$ with $p+q=2$. This splitting, the intersection form and the cone of all Kähler classes are $\operatorname{Aut}(X)^{*}$-invariant (see [28]). Hence, it would be too much to require $\varphi$ to be an isomorphism.

There are only countably many distinct groups $\operatorname{Aut}(X)^{*}$. This follows from the fact that, up to isomorphism, there are only countably many pairwise non-isomorphic extensions of $\mathbf{Q}$ which are finitely generated. On the opposite, for every integer $n \geq 4$, there are uncountably many, pairwise non-isomorphic, subgroups of $\mathrm{GL}_{n}(\mathbf{Z})$. Thus a counting argument shows that most subgroups $\Gamma$ of $\mathrm{GL}_{n}(\mathbf{Z})$ are not realizable as groups of automorphisms. On the other hand, there are only countably many subgroups of $\mathrm{GL}_{n}(\mathbf{Z})$ which are finitely generated.

Question 2.5. Which subgroups of $\mathrm{GL}_{n}(\mathbf{Z})$ are abstractly (resp. strongly) realizable as groups of automorphisms ? Is every finitely generated subgroup $\Gamma \subset \mathrm{GL}_{n}(\mathbf{Z})$ abstractly (resp. strongly) realizable as a group of automorphisms ?

Recently, Lesieutre proved that if $\mathbf{k}$ is a field of characteristic 0 , or a field which is not algebraic over its prime field, then there is a smooth, 6-dimensional projective variety $X$ over $\mathbf{k}$ such that $\operatorname{Aut}(X)^{0}$ is trivial and $\operatorname{Aut}(X)^{*}$ is not finitely generated (see [34] and [20] for another example). This shows that it is somewhat artificial to assume that $\Gamma$ is finitely generated. 
Remark 2.6. The following problem remains open: does there exist a rational surface $S$ such that $\operatorname{Aut}(S)$ is discrete but not finitely generated ? More precisely, can one find such an example which is a minimal model for the pair $(S, \operatorname{Aut}(S))$, meaning that if $\pi: S \rightarrow S^{\prime}$ is a birational morphism and $\pi \circ \operatorname{Aut}(S) \circ \pi^{-1} \subset \operatorname{Aut}\left(S^{\prime}\right)$ then $\pi$ is an isomorphism ?

In Question 2.5, the dimension of $X$ is not specified; indeed, $\operatorname{dim}(X)$ must a priori be large with respect to $n$. Changing viewpoint, one may fix the dimension, or fix the type of variety one considers, and try to find constraints on the subgroups $\operatorname{Aut}(X)^{*} \subset \mathrm{GL}\left(H^{2}(X ; \mathbf{Z})\right)$.

Question 2.7. Fix a dimension $d \geq 2$. Define $G^{0}(X)$ as the neutral component of the Zariski closure of $\operatorname{Aut}(X)^{*}$ in $\mathrm{GL}\left(H^{2}(X ; \mathbf{R})\right)$. What kind of linear algebraic groups $G^{0}(X)$ do we obtain in this way, when $X$ runs over all possible Calabi-Yau varieties of dimension $d$ ?

Again, by Remark 2.1, the group $\operatorname{Aut}(X)$ may be replaced by the group Psaut $(X)=$ $\operatorname{Bir}(X)$ and $H^{2}(X ; \mathbf{R})$ by $\mathrm{N}^{1}(X) \otimes_{\mathbf{Z}} \mathbf{R}$ in this question. For a nice example, see [13]. For complex projective K3 surfaces, the groups $G^{0}(X)$ that one gets are connected components of $\mathrm{SO}_{1, \mathrm{~m}}(\mathbf{R})$ for some $m \leq 19$, or abelian groups $\mathbf{R}^{m}$ of rank $m \leq 18$. This comes from Hodge index theorem.

Question 2.5 is stated for Calabi-Yau manifolds because they form one of the most interesting classes of examples, but it may be stated for other classes, for instance for rational varieties. And instead of looking at the action of $\operatorname{Aut}(X)$ on $H^{2}(X ; \mathbf{R})$, one may also consider its action on every Dolbeault subgroup $H^{p, q}(X ; \mathbf{C})$ (see $[11,15,47])$.

2.4. Real algebraic variation. Consider now a smooth real projective variety $X_{\mathbf{R}}$ of dimension $d$. Assume that $X(\mathbf{R})$ is non-empty, and fix one of the connected components $S \subset X(\mathbf{R})$ (for the euclidean topology); then $S$ is a closed connected manifold of dimension $d$. The automorphisms of $X$ which are defined over $\mathbf{R}$ form a subgroup $\operatorname{Aut}\left(X_{\mathbf{R}}\right)$ of $\operatorname{Aut}\left(X_{\mathbf{C}}\right)$. A finite index subgroup $\operatorname{Aut}\left(X_{\mathbf{R}} ; S\right)$ of $\operatorname{Aut}\left(X_{\mathbf{R}}\right)$ fixes $S$, and we get a restriction morphism

$$
\operatorname{Aut}\left(X_{\mathbf{R}} ; S\right) \rightarrow \operatorname{Diff}^{\infty}(S)
$$

where $\operatorname{Diff}{ }^{\infty}(S)$ is the group of $\mathcal{C}^{\infty}$-diffeomorphisms of $S$. Denoting by $\operatorname{Mod}(S)$ the modular group of $S$, i.e. the group of connected components of $\operatorname{Diff}^{\infty}(S)$ (see [25]), one gets a homomorphism

$$
\alpha_{S}: \operatorname{Aut}\left(X_{\mathbf{R}} ; S\right) \rightarrow \operatorname{Mod}(S) .
$$

What can be said on the image of this homomorphism?

The best is to start with surfaces. First, there are explicit examples of automorphisms $f$ of rational and K3 surfaces for which the mapping class $\alpha_{S}(f)$ is interesting (see $[1,41]$ ). Second, Kollár and Mangolte obtained the following beautiful result. Fix any smooth real surface $X_{\mathbf{R}}$, which is rational (over $\mathbf{R}$ ). Then, consider the subgroup $\operatorname{Bir}^{\infty}\left(X_{\mathbf{R}}\right)$ of $\operatorname{Bir}\left(X_{\mathbf{R}}\right)$ defined by the following property: $f$ is in $\operatorname{Bir}^{\infty}\left(X_{\mathbf{R}}\right)$ if and only if $f$ and its inverse $f^{-1}$ have no real indeterminacy point - all its indeterminacy points come in complex conjugate pairs. By restriction to $X(\mathbf{R})$, each 
element $f$ of $\operatorname{Bir}^{\infty}\left(X_{\mathbf{R}}\right)$ provides a diffeomorphism of $X(\mathbf{R})$, and therefore a mapping class $\alpha_{X(\mathbf{R})}(f) \in \operatorname{Mod}(X(\mathbf{R}))$. Kollár and Mangolte proved in [32] that this homomorphism $\operatorname{Bir}^{\infty}\left(X_{\mathbf{R}}\right) \rightarrow \operatorname{Mod}(X(\mathbf{R}))$ is surjective.

Question 2.8. Does there exist a real projective K3 surface $X$ such that (i) $X(\mathbf{R})$ is connected and of genus $g \geq 2$ and (ii) the image of $\alpha_{X(\mathbf{R})}: \operatorname{Aut}\left(X_{\mathbf{R}}\right) \rightarrow \operatorname{Mod}(X(\mathbf{R}))$ is surjective?

I suspect that the answer to this question is negative. Indeed, there may exist a mapping class $\psi$ of the closed, orientable surface of genus 2 with the following property: given any automorphism $f$ of a real K3 surface $X_{\mathbf{R}}$ with an $f$-invariant connected component $S \subset X(\mathbf{R})$ of genus $2, \alpha_{S}(f)$ is not conjugate to $\psi$.

2.5. Dynamical degrees. Consider an automorphism $f$ of a smooth complex projective variety $X$. The characteristic polynomial $\chi_{f}(t)$ of

$$
f^{*}: H^{2}(X ; \mathbf{Z}) \rightarrow H^{2}(X ; \mathbf{Z})
$$

is an element of $\mathbf{Z}[t]$; it is monic, and its constant term is \pm 1 . Denote by $\lambda_{1}(f)$ the largest absolute value of a root of $\chi_{f}(t)$. The invariance of the Hodge decomposition and of the Kähler cone implies that $\lambda_{1}(f)$ is in fact one of the roots of $\chi_{f}(t)$ and, as such, is an algebraic integer. This number $\lambda_{1}(f)$ is called the first dynamical degree of $f$ (subsequent dynamical degrees are obtained by looking at the action of $f$ on the groups $H^{p, p}(X ; \mathbf{R})$ with $\left.p>1\right)$. Now, fix the dimension $d$ of the variety, and define the following set of real integers:

$$
\mathfrak{S}_{1}(d)=\left\{\lambda_{1}(f) ; f \in \operatorname{Aut}(X) \text { for } X \text { smooth, projective, of dimension } d\right\} .
$$

In dimension $1, \mathfrak{S}_{1}(1)=\{1\}$, but in dimension $d \geq 2, \mathfrak{S}_{1}(d)$ is an infinite countable set of algebraic integers. In dimension 2 , the Hodge index theorem shows that $\mathfrak{S}_{1}(2) \backslash$ $\{1\}$ contains only reciprocal quadratic integers and Salem numbers; and its first derive set is non empty: for example the golden mean is an increasing limit of elements of $\mathfrak{S}_{1}(2)$ (see [19]). A deeper result says that every strictly decreasing sequence of elements of $\mathfrak{S}_{1}(2)$ is finite; in particular, given any $\alpha \in \mathfrak{S}_{1}(2)$, there is a real number $\epsilon(\alpha)>0$ such that $] \alpha, \alpha+\epsilon(\alpha)\left[\right.$ does not intersect $\mathfrak{S}_{1}(2)$ (see $\left.[38,40,3]\right)$. In particular, many Salem numbers are not contained in $\mathfrak{S}_{1}(2)$. The only general constraint known on the elements of $\mathfrak{S}_{1}(3)$ is due to Lo Bianco: if $\alpha \in \mathfrak{S}_{1}(3)$, there are at most 6 distinct moduli $\left|\alpha_{i}\right|$ for the Galois conjugates $\alpha_{i}$ of $\alpha$ (whatever the degree of the algebraic integer $\alpha$, see [37]).

Question 2.9. Is $\mathfrak{S}_{1}(3)$ dense in $[1,+\infty[$ ? Or, on the contrary, does there exist $\epsilon>0$ such that $\left.\left.\mathfrak{S}_{1}(3) \cap\right] 1,1+\epsilon\right]$ is empty?

In this question, on may replace $\mathfrak{S}_{1}(3)$ by the set of dynamical degrees of all birational transformations of projective threefolds; here, by definition, the first dynamical degree of a birational transformation $f \in \operatorname{Bir}(X)$ is defined by the limit

$$
\lambda_{1}(f)=\lim _{n \rightarrow+\infty}\left(\left(f^{n}\right)^{*}(H) \cdot H^{d-1}\right)^{1 / n}
$$

where $H$ is some hyperplane section of $X, d$ is the dimension of $X$, and the integer $\left(f^{n}\right)^{*}(H) \cdot H^{d-1}$ is the intersection product of the total transform $\left(f^{n}\right)^{*}(H)$ with 
$d-1$ copies of $H$. This limit exists and does not depend on $H$ (see $[21,16,43]$, and Section 3.3 below).

\section{Dynamics with LOW COMplexity}

3.1. Entropy. Given a continuous transformation $g$ of a compact metric space $M$, the topological entropy $\mathrm{h}_{t o p}(g ; M)$ is a measure of the complexity of the dynamics of $g: M \rightarrow M$. To define it, fix a scale $\epsilon>0$ at which you observe the dynamics. Given a period of observation $N \in \mathbf{Z}_{+}$, count the maximum number of orbits $\left(x, g(x), \ldots, g^{N-1}(x)\right)$ that are pairwise distinct at scale $\epsilon$, the orbit of $x$ being distinguishable from the orbit of $y$ if the distance from $g^{k}(x)$ to $g^{k}(y)$ is larger than $\epsilon$ for at least one time $0 \leq k<N$. This number of orbits $\operatorname{Orb}(N ; \epsilon)$ typically grows exponentially fast with $N$; thus, one defines $h(g ; \epsilon)$ as the supremum limit of $\frac{1}{N} \log (\operatorname{Orb}(N ; \epsilon))$ and the entropy $\mathrm{h}_{t o p}(g ; M)$ of $g$ as the limit of $h(g ; \epsilon)$ as $\epsilon$ goes to 0 , i.e. as our observations become arbitrarily accurate.

Topological entropies are hard to estimate for continuous or smooth maps of manifolds. But the topological entropy of an endomorphism $f$ of a smooth complex projective variety $X$ is equal to the logarithm of the spectral radius of $f^{*}: H^{*}(X ; \mathbf{C}) \rightarrow$ $H^{*}(X ; \mathbf{C})$ (the action of $f$ on the cohomology). This wonderful result is due to Gromov and Yomdin (see [30, 29, 46], and [21] for an upper bound when $f$ is a rational map). For instance, the entropy is invariant under deformation: if $F$ is an automorphism of a variety $\mathcal{X}$ that preserves a fibration $\pi: \mathcal{X} \rightarrow B$ with smooth projective fibers and the fibration is locally trivial topologically, then the automorphisms induced by $F$ on the fibers of $\pi$ have the same entropy.

Endomorphisms with positive entropy have been studied in detail, with special focus on endomorphisms of the projective space $\mathbb{P}_{\mathbf{C}}^{k}$ and automorphisms of surfaces. We refer to $[9,22]$ for survey papers on the subject. Here, we consider the opposite edge of the spectrum: instead of looking at automorphisms with chaotic dynamics, we ask for a description of automorphisms with dynamics of low complexity.

3.2. Invariant fibrations. Now consider an automorphism $f$ of a smooth complex projective variety $X$ with entropy equal to 0 . Then, by the Gromov-Yomdin theorem, the eigenvalues of $f^{*} \in \mathrm{GL}\left(H^{*}(X ; \mathbf{Z})\right)$ all have modulus 1 , and being algebraic integers, they must be roots of unity. Changing $f$ in a positive iterate, we may assume that $f^{*}$ is unipotent: all its eigenvalues are equal to 1 .

If $f^{*}$ is the identity, then some further iterate is contained in $\operatorname{Aut}(X)^{0}$ (see Section 2.1). The dynamics of such an automorphism is well understood. Thus, one may assume that $f^{*}$ is non-trivial and unipotent; equivalently, the sequence $\left\|\left(f^{n}\right)^{*}\right\|$ grows polynomially quickly with $n$, as $n^{k}$ for some $k \geq 1$.

When $\operatorname{dim}(X)=2$, Gizatullin proved the following: if $f^{*}$ is unipotent and $\neq \mathrm{Id}$, then $f$ preserves a (singular) fibration by curves of genus 1 and the growth of $\left\|\left(f^{n}\right)^{*}\right\|$ is quadratic (see $[27,19,6]$ ). For instance, if $\pi: X \rightarrow B$ is a genus 1 fibration of the surface $X$ with two sections, then the translations along the fibers that map the first section to the second one determine an algebraic transformation of $X$ : this is often an automorphism such that $\left\|\left(f^{n}\right)^{*}\right\|$ grows quadratically. The following question asks whether Gizatullin's classification can be extended to higher dimension. 
Question 3.1. Let $X$ be a smooth complex projective variety of dimension 3. Let $f$ be an automorphism of $X$, such that the linear transformation $f^{*} \in \mathrm{GL}\left(H^{*}(X ; \mathbf{C})\right)$ is non-trivial and unipotent. Does $f$ permute the fibers of a non-trivial meromorphic fibration $\pi: X \rightarrow B$ ?

By "non-trivial meromorphic fibration", I mean that $0<\operatorname{dim}(B)<\operatorname{dim}(X)$ and $\pi$ is a dominant rational map; and $f$ permutes the fibers of $\pi$ if there is a birational transformation $f_{B}$ of $B$ such that $\pi \circ f=f_{B} \circ \pi$.

When $f$ is an automorphism of a smooth complex projective variety of dimension 3 and $f^{*}$ is a non-trivial unipotent matrix, then $\left\|\left(f^{*}\right)^{n}\right\|$ grows like $n^{k}$ with $k \in$ $\{2,4\}$ (see [37]). But beside this general statement, not much is known.

One of the first examples to look at is the case of Calabi-Yau varieties. So, let $f$ be an automorphism of a Calabi-Yau variety $X$ of dimension 3 , with a non-trivial unipotent action on the cohomology. Consider a kähler form $\kappa$ on $X$, with cohomology class $[\kappa] \in H^{2}(X ; \mathbf{R})$; then $n^{-k}\left(f^{n}\right)^{*}[\kappa]$ converges towards an $f$-invariant nef class. Is this class related to an $f$-invariant fibration on $X$ ? This is a version of the abundance conjecture (see [33]), precisely in a case which is not solved yet, but with the additional presence of an automorphism.

3.3. Degree growth. If an automorphism satisfies $\lambda_{1}(f)=1$, then an iterate of $f^{*}$ is unipotent, and $\left\|\left(f^{n}\right)^{*}\right\|$ grows polynomially with $n$. Now, consider a birational transformation $f: X \rightarrow X$ of a complex projective variety $X$. Fix a polarization $H$ of $X$ and define the degree of $f$ with respect to $H$ by the intersection product

$$
\operatorname{deg}_{H}(f)=f^{*}(H) \cdot H^{d-1}
$$

where $d$ is the dimension of $X$ and $f^{*}(H)$ is the total transform of $H$ by $f^{-1}$. Changing $H$ into another polarization $H^{\prime}$, the notion of degree is only perturbed by a bounded multiplicative error: there exists a positive constant $c$ such that

$$
\frac{1}{c} \operatorname{deg}_{H^{\prime}}(f) \leq \operatorname{deg}_{H}(f) \leq c \operatorname{deg}_{H^{\prime}}(f)
$$

for all $f \in \operatorname{Bir}(X)$ (see $[21,16,43]$ ). When $X=\mathbb{P}_{\mathbf{C}}^{d}$ and $H \subset \mathbb{P}_{\mathbf{C}}^{d}$ is a hyperplane, then $\operatorname{deg}_{H}(f)$ is just the degree of the polynomials that define $f$ in homogeneous coordinates (see Section 1.2.1). Instead of assuming that the entropy of $f$ is zero, one may now assume that $\lambda_{1}(f)=1$, i.e. that $\operatorname{deg}_{H}\left(f^{n}\right)$ does not grow exponentially fast with $n$.

Question 3.2. Let $X$ be a smooth projective variety of dimension $d$, together with a polarization $H$. Let $f$ be a birational transformation of $X$ with $\lambda_{1}(f)=1$. Does the sequence $\operatorname{deg}_{H}\left(f^{n}\right)$ grow polynomially ? If not bounded, does this sequence grow at least linearly?

In other words, can one construct a birational transformation $f$, say, of $\mathbb{P}_{\mathbf{C}}^{3}$, such that the sequence $\operatorname{deg}_{H}\left(f^{n}\right)$ grows like $\exp (\sqrt{n})$, or like $n^{2} \log (n)$, or like $n^{1 / 3}$ ? This is already an open problem for polynomial automorphisms of the affine space $\mathbb{A}_{\mathbf{C}}^{3}$. One may also ask, as in Question 3.1, whether the equality $\lambda_{1}(f)=1$ implies the existence of a non-trivial invariant fibration. While these questions are fully understood in 
dimension 2 (see $[8,10,19,27]$ ) almost nothing is known in higher dimension (see [44], as well as $[18,36]$ for interesting examples).

This type of question is, in fact, directly related to the algebraic structure of the group $\operatorname{Bir}(X)$. For instance, one says that an element $f$ of $\operatorname{Bir}(X)$ is distorted if there exists a finite set $S \subset \operatorname{Bir}(X)$ such that the $n$-th iterate $f^{n}$ is a product $g_{1} \circ \cdots \circ g_{\ell(n)}$ of elements $g_{i} \in S$ with $\liminf (\ell(n) / n)=0$ (see [5]). Distorted elements satisfy $\lambda_{1}(f)=1$. In dimension 2 , an element $f$ of $\operatorname{Bir}\left(\mathbb{P}_{\mathbf{C}}^{2}\right)$ is distorted if and only if some positive iterate $f^{N}$ is conjugate to an element of $\operatorname{Aut}\left(\mathbb{P}_{\mathbf{C}}^{2}\right)$. Any progress on Questions 3.1 and 3.2 would certainly help classify distorted elements in higher dimension.

3.4. Minimal transformations. A continuous transformation $g$ of a compact metric space $M$ is minimal if each of its orbits are dense; equivalently, the only $g$ invariant closed subsets are $M$ and the empty set. For an algebraic example, consider a complex torus $A=\mathbf{C}^{k} / \Lambda$, with $\Lambda$ a cocompact lattice in $\mathbf{C}^{k}$; then, every totally irrational translation on $A$ is minimal. For another example, take $k=2$ and $\Lambda=\Lambda_{0} \times \Lambda_{0}$, with $\Lambda_{0}$ a lattice in $\mathbf{C}$. Choose a point $a$ in $\mathbf{C} / \Lambda_{0}$ such that each orbit of $x \mapsto x+a$ is dense in $\mathbf{C} / \Lambda_{0}$, and consider the following automorphism of $\mathbf{C}^{2} / \Lambda$ :

$$
f(x, y)=(x+a, y+x) \quad \bmod (\Lambda) .
$$

Furstenberg shows in [26] that every orbit of $f$ is dense in the abelian surface $\mathbf{C}^{2} / \Lambda$. In fact, Furstenberg proves more: $f$ preserves the Haar measure on the torus and this is the unique $f$-invariant probability measure. Thus, there are minimal automorphisms with no positive iterate in $\operatorname{Aut}(X)^{0}$ (in Furstenberg's example, the action of $f$ on the cohomology is non-trivial and unipotent).

A minimal automorphism of a curve is a translation on an elliptic curve. In dimension 2, one also proves easily that any complex projective surface $X$ with a minimal automorphism is an abelian surface. It would be great to get a similar result in higher dimension:

Question 3.3. Let $X$ be a complex projective variety of dimension 3. Suppose that there is a minimal automorphism $f$ on $X$. Is $X$ an abelian variety ?

If $f$ is minimal it does not have any periodic orbit. With the Lefschetz formula and Gromov-Yomdin theorem, one sees that the entropies of minimal automorphisms of complex projective varieties vanish. Now, if $X$ has dimension 3 , the holomorphic Lefschetz formula says that $h^{1,0}(X)>0$ or $h^{3,0}(X)>0$. If $h^{1,0}(X)>0$, one may use the Albanese morphism to reduce the complexity of the dynamics of $f$ (see [7]). If $h^{3,0}(X)>0$, there is an $f$-invariant holomorphic 3 -form on $X$; since $f$ does not preserve any strict Zariski closed set (by minimality), this form does not vanish. Thus, the most interesting case is when $X$ is a Calabi-Yau variety of dimension 3 with Euler characteristic equal to 0, and the action of $f^{m}$ on the cohomology is unipotent for some $m>0$ (as in $\S 3.2$ ).

\subsection{Fatou components and real algebraic varieties.}


3.5.1. Fatou components on K3 surfaces. Consider an automorphism $f$ of a K3 surface $X$. Assume that the topological entropy of $f$ on the complex surface $X(\mathbf{C})$ is positive: $\mathrm{h}_{t o p}(f ; \mathbf{C})=\log \left(\lambda_{1}(f)\right)>0$ where $\lambda_{1}(f)$ is, by the Gromov-Yomdin theorem, the largest eigenvalue of $f$ on the cohomology of $X(\mathbf{C})$. In that case, $f$ has infinitely many periodic points, and those points equidistribute toward an $f$-invariant probability measure $\mu_{f}$ (see $\left.[6,9,24]\right)$. More precisely, denote by $\operatorname{Per}(f ; N)$ the set of isolated periodic points of $f$ or period $N$. Then, the sequence of probability measures

$$
\frac{1}{|\operatorname{Per}(f ; N)|} \sum_{z \in \operatorname{Per}(f ; N)} \delta_{z}
$$

converges toward an $f$-invariant probability measure $\mu_{f}$ with interesting dynamical properties (this is the unique measure of maximal entropy of $f$ ). The same property holds if one replaces $\operatorname{Per}(f ; N)$ by the set of saddle periodic points of period $N$, i.e. by points $x \in X(\mathbf{C})$ such that $f^{N}(x)=x$ and the differential $D f_{x}^{N}$ has two eigenvalues $\alpha$ and $\beta$ with

$$
|\alpha|>1>|\beta| ;
$$

indeed, the number of isolated periodic points and of saddle periodic points grow at the same speed, namely like $\lambda_{1}(f)^{N}$.

The measure $\mu_{f}$ is, in general, singular with respect to the Lebesgue measure on $X(\mathbf{C})$. If $\mu_{f}$ is absolutely continuous with respect to the Lebesgue measure, then $(X, f)$ is a Kummer example: this means that $X$ is a quotient of an abelian surface $A$ and $f$ is induced by a linear automorphism of $A$ (see [12]).

But not much is known about the support of the measure $\mu_{f}$. There may a priori exist a region $\mathcal{U} \subset X(\mathbf{C})$ which is $f$-invariant and on which the dynamics of $f$ is far from being chaotic. More precisely, define the Fatou set Fat $(f)$ as follows: $x \in X(\mathbf{C})$ is in the Fatou set if there is an open neighborhood $\mathcal{V}$ of $x$ such that the sequence of iterates $\left(f^{m}\right)_{m \in \mathbf{Z}}$ forms a normal family on $\mathcal{V}$, in the sense of Montel. This determines an $f$-invariant open subset $\operatorname{Fat}(f) \subset X(\mathbf{C})$ on which $\mu_{f}$ vanishes. It is not known yet whether the Fatou set is always empty for automorphisms of complex projective K3 surfaces with positive entropy. There are examples of non-empty Fatou sets on non-projective K3 surfaces and rational surfaces (see [39, 1]).

Question 3.4. Does there exist a complex projective K3 surface $X$ with an automorphism $f: X \rightarrow X$ such that the topological entropy of $f$ is positive and the Fatou set of $f$ is not empty?

While the theory of closed positive currents, the Hodge theory, and the Pesin theory of smooth dynamical systems may be combined to study the chaotic part of the dynamics from a stochastic viewpoint (see $[2,6,9,17,23]$ for instance), not much is known concerning the topological properties of the dynamics, such as the existence of non-empty Fatou components or dense orbits. Thus, Question 3.4 is at the borderline of our knowledge.

3.5.2. Dynamics on real K3 surfaces. Now, add to the hypotheses that $X$ and $f$ are defined over the field of real numbers $\mathbf{R}$, and $X(\mathbf{R})$ is not empty. Fix a connected component $S$ of $X(\mathbf{R})$, and replace $f$ by some positive iterate so that $f$ now 
preserves $S$. We obtain two dynamical systems: the complex dynamics given by $f: X(\mathbf{C}) \rightarrow X(\mathbf{C})$, and the real dynamics given by $f: S \rightarrow S$. The topological entropy of $f$ on $S$ is bounded above by the topological entropy of $f$ on $X(\mathbf{C})$. For rational surfaces, it may happen that these two numbers are equal (and positive, see [1]); in that case most periodic points of $f$ are indeed contained in $X(\mathbf{R})$. For K3 surfaces, there is no example yet with

$$
\mathrm{h}_{t o p}(f ; X(\mathbf{R}))>\frac{1}{2} \mathrm{~h}_{t o p}(f ; X(\mathbf{C})) .
$$

At the opposite edge of the possibilities, there is no known example for which $\mathrm{h}_{\text {top }}(f ; X(\mathbf{C}))$ is positive while $\mathrm{h}_{\text {top }}(f ; S)=0$ for some connected component of $X(\mathbf{R})$ (see $[42])$.

Question 3.5. Does there exist a real projective K3 surface $X_{\mathbf{R}}$ with an automorphism $f: X_{\mathbf{R}} \rightarrow X_{\mathbf{R}}$ such that $\lambda_{1}(f)>1, X(\mathbf{R})$ is not empty, and one of the following propery occurs

(1) the entropy of $f$ on $X(\mathbf{R})$ is equal to the entropy of $f$ on $X(\mathbf{C})$ ?

(2) the entropy of $f$ on some component $S$ of $X(\mathbf{R})$ vanishes ?

(3) a connected component $S$ of $X(\mathbf{R})$ is contained in the Fatou set of $f$ ?

Of course, a positive answer to the third of these questions would imply a positive answer to the second question and to Question 3.4. To obtain a positive answer to the first, it would be sufficient to find a component $S \subset X(\mathbf{R})$ which is $f$ invariant and such that the mapping class $\alpha_{S}(f)$ has a stretching factor equal to $\lambda_{1}(f)$ on the fundamental group $\pi_{1}(S)$ (see [25]); thus, Question 3.5 is also related to Section 2.4.

\section{REFERENCES}

[1] Eric Bedford and Kyounghee Kim. Dynamics of rational surface automorphisms: linear fractional recurrences. J. Geom. Anal., 19(3):553-583, 2009.

[2] Eric Bedford, Mikhail Lyubich, and John Smillie. Polynomial diffeomorphisms of $\mathbf{C}^{2}$. IV. The measure of maximal entropy and laminar currents. Invent. Math., 112(1):77-125, 1993.

[3] Jérémy Blanc and Serge Cantat. Dynamical degrees of birational transformations of projective surfaces. J. Amer. Math. Soc., 29(2):415-471, 2016.

[4] Michel Brion. On automorphisms and endomorphisms of projective varieties. In Automorphisms in birational and affine geometry, volume 79 of Springer Proc. Math. Stat., pages 59-81. Springer, Cham, 2014.

[5] Danny Calegari and Michael H. Freedman. Distortion in transformation groups. Geom. Topol., 10:267-293, 2006. With an appendix by Yves de Cornulier.

[6] Serge Cantat. Dynamique des automorphismes des surfaces K3. Acta Math., 187(1):1-57, 2001.

[7] Serge Cantat. Quelques aspects des systèmes dynamiques polynomiaux: existence, exemples, rigidité. In Quelques aspects des systèmes dynamiques polynomiaux, volume 30 of Panor. Synthèses, pages 13-95. Soc. Math. France, Paris, 2010.

[8] Serge Cantat. The Cremona group in two variables. In European Congress of Mathematics, pages 211-225. Eur. Math. Soc., Zürich, 2013.

[9] Serge Cantat. Dynamics of automorphisms of compact complex surfaces. In Frontiers in complex dynamics, volume 51 of Princeton Math. Ser., pages 463-514. Princeton Univ. Press, Princeton, NJ, 2014.

[10] Serge Cantat. The Cremona groups. Proceedings of the 2015 Summer Institute in Algebraic Geometry, to appear:1-48, 2015. 
[11] Serge Cantat and Igor Dolgachev. Rational surfaces with a large group of automorphisms. $J$. Amer. Math. Soc., 25(3):863-905, 2012.

[12] Serge Cantat and Christophe Dupont. Automorphisms of surfaces: Kummer rigidity and measure of maximal entropy. preprint, pages 1-68, 2015.

[13] Serge Cantat and Keiji Oguiso. Birational automorphism groups and the movable cone theorem for Calabi-Yau manifolds of Wehler type via universal Coxeter groups. Amer. J. Math., 137(4):1013-1044, 2015.

[14] Serge Cantat and Junyi Xie. Algebraic actions of discrete groups: The p-adic method. preprint, pages $1-52,2015$.

[15] Serge Cantat and Abdelghani Zeghib. Holomorphic actions, Kummer examples, and Zimmer program. Ann. Sci. Éc. Norm. Supér. (4), 45(3):447-489, 2012.

[16] Nguyen-Bac Dang. Degrees of iterates of rational maps on normal projective varieties. preprint, arXiv:1701.07760, pages 1-46, 2017.

[17] Henry De Thélin and Tien-Cuong Dinh. Dynamics of automorphisms on compact Kähler manifolds. Adv. Math., 229(5):2640-2655, 2012.

[18] Julie Déserti. Degree growth of polynomial automorphisms and birational maps: some examples. Eur. J. Math., to appear, pages 1-10, 2017.

[19] Jeffrey Diller and Charles Favre. Dynamics of bimeromorphic maps of surfaces. Amer. J. Math., 123(6):1135-1169, 2001.

[20] Tien-Cuong Dinh and Keiji Oguiso. A surface with discrete and non-finitely generated automorphism group. preprint, arXiv:1710.07019, pages 1-11, 2017.

[21] Tien-Cuong Dinh and Nessim Sibony. Une borne supérieure pour l'entropie topologique d'une application rationnelle. Ann. of Math. (2), 161(3):1637-1644, 2005.

[22] Tien-Cuong Dinh and Nessim Sibony. Dynamics in several complex variables: endomorphisms of projective spaces and polynomial-like mappings. In Holomorphic dynamical systems, volume 1998 of Lecture Notes in Math., pages 165-294. Springer, Berlin, 2010.

[23] Tien-Cuong Dinh and Nessim Sibony. Super-potentials for currents on compact Kähler manifolds and dynamics of automorphisms. J. Algebraic Geom., 19(3):473-529, 2010.

[24] Romain Dujardin. Laminar currents and birational dynamics. Duke Math. J., 131(2):219-247, 2006.

[25] Benson Farb and Dan Margalit. A primer on mapping class groups, volume 49 of Princeton Mathematical Series. Princeton University Press, Princeton, NJ, 2012.

[26] Harry Furstenberg. Strict ergodicity and transformation of the torus. Amer. J. Math., 83:573601, 1961.

[27] Marat H. Gizatullin. Rational G-surfaces. Izv. Akad. Nauk SSSR Ser. Mat., 44(1):110-144, 239, 1980.

[28] Phillip Griffiths and Joseph Harris. Principles of algebraic geometry. Wiley-Interscience [John Wiley \& Sons], New York, 1978. Pure and Applied Mathematics.

[29] Mikhaïl Gromov. Entropy, homology and semialgebraic geometry. Astérisque, (145-146):5, 225240, 1987. Séminaire Bourbaki, Vol. 1985/86.

[30] Mikhail Gromov. On the entropy of holomorphic maps. Enseign. Math. (2), 49(3-4):217-235, 2003.

[31] Alexander Grothendieck. Techniques de construction et théorèmes d'existence en géométrie algébrique. IV. Les schémas de Hilbert. In Séminaire Bourbaki, Vol. 6, pages Exp. No. 221, 249-276. Soc. Math. France, Paris, 1995.

[32] János Kollár and Frédéric Mangolte. Cremona transformations and diffeomorphisms of surfaces. Adv. Math., 222(1):44-61, 2009.

[33] Vladimir Lazić, Keiji Oguiso, and Thomas Peternell. Nef line bundles on Calabi-Yau threefolds, i. preprint, arXiv:1601.01273v2, pages 1-37, 2016.

[34] John Lesieutre. A projective variety with discrete, non-finitely generated automorphism group. Inventiones Math., to appear, pages 1-13, 2017.

[35] David I. Lieberman. Compactness of the Chow scheme: applications to automorphisms and deformations of Kähler manifolds. In Fonctions de plusieurs variables complexes, III (Sém. 
François Norguet, 1975-1977), volume 670 of Lecture Notes in Math., pages 140-186. Springer, Berlin, 1978.

[36] Jan-Li Lin. On degree growth and stabilization of three-dimensional monomial maps. Michigan Math. J., 62(3):567-579, 2013.

[37] Federico Lo Bianco. Bornes sur les degrés dynamiques d'automorphismes de variétés kählériennes de dimension 3. C. R. Math. Acad. Sci. Paris, 352(6):515-519, 2014.

[38] Curtis T. McMullen. Coxeter groups, Salem numbers and the Hilbert metric. Publ. Math. Inst. Hautes Études Sci., (95):151-183, 2002.

[39] Curtis T. McMullen. Dynamics on K3 surfaces: Salem numbers and Siegel disks. J. Reine Angew. Math., 545:201-233, 2002.

[40] Curtis T. McMullen. Dynamics on blowups of the projective plane. Publ. Math. Inst. Hautes Études Sci., (105):49-89, 2007.

[41] Arnaud Moncet. Un exemple de groupe d'automorphismes d'une surface k3 réelle. Master 2, https://perso.univ-rennes1.fr/serge.cantat/Documents/Moncet-M2.pdf, pages 1-31, 2008.

[42] Arnaud Moncet. Real versus complex volumes on real algebraic surfaces. Int. Math. Res. Not. IMRN, (16):3723-3762, 2012.

[43] Tuyen Trung Truong. Relative dynamical degrees of correspondences over a field of arbitrary characteristic. preprint, arXiv:1701.07760, pages 1-41, 2016.

[44] Christian Urech. Remarks on the degree growth of birational transformations. Math. Research Letters, to appear, pages 1-12, 2017.

[45] Jörg Winkelmann. Realizing connected Lie groups as automorphism groups of complex manifolds. Comment. Math. Helv., 79(2):285-299, 2004.

[46] Yosef Yomdin. Volume growth and entropy. Israel J. Math., 57(3):285-300, 1987.

[47] De-Qi Zhang. Algebraic varieties with automorphism groups of maximal rank. Math. Ann., 355(1):131-146, 2013.

Univ Rennes, CNRS, IRMAR - UMR 6625, F-35000 Rennes, France

E-mail address: serge.cantat@univ-rennes1.fr 